\title{
3. The relationship between the population's socio-economic status and walkability measures: the context of the Lisbon metropolitan area
}

\author{
Mauro F. Pereira, Paula Santana and David S. \\ Vale
}

\subsection{INTRODUCTION}

The socio-economic status (SES) of an individual is crucial to define the most significant aspects of individual life (Marmot et al., 2012). Aspects such as employment, education and income shape lifestyle and access to different opportunities (Marmot et al., 2012). Population distribution in the cities is made according to their SES, reflecting the wealth of these places. A walkable environment with better accessibility by different transport modes results in higher property values, which reduces the diverse population status with income capacity to access these places (Gilderbloom et al., 2015). Property value defines the ability of different socio-economic groups to acquire or rent houses (Drewnowski et al., 2014; Gilderbloom et al., 2015). Property value is influenced not only by the features of the property itself, but also by different built environment characteristics; namely, neighbourhood perception, the streetscape's qualities, and mostly by accessibility (Carr et al., 2010). Accessibility is crucial to individuals' daily activities, access to work, access to healthcare, and so on. Accessibility to places affects differently individuals of different socio-economic groups (Delbosc and Currie, 2011; Lucas, 2012). Low accessibility has a high impact in the most deprived groups because their reduced income decreases the options available to access different activities. For example, higher income socio-economic groups have the power to suppress the scarcity of walking infrastructures and public transport access by using several individual modes (Delbosc and Currie, 2011).

The Lisbon Metropolitan Area (LMA) is a particularly interesting context because it has very different urban and demographic contexts, a result of its 
dimension and changes across time. However, there is little knowledge about the relationship between socio-economic groups' distribution and walkable characteristics. The goal of the chapter is to understand the distribution and relationship between walkable characteristics and socio-economic groups across the LMA, distinguishing areas according to their travel time to the city centre. To achieve this goal, different indicators used to measure walkability were tested and correlated with SES indicators.

\subsection{BACKGROUND}

Health and wellbeing determinants define an ecological model where individual characteristics are in the centre of this model, but context spheres also influence individual health and wellbeing (Barton and Grant, 2006). Individual socio-demographics play a crucial role in health and wellbeing. However, the sphere of closer context at the level of the bespoke neighbourhood had influences on these individual characteristics, allowing healthier lifestyles. The spatial built environment characteristics define the access level of different opportunities and where transport options are crucial to access these opportunities (Geurs and van Wee, 2004). Transport options have different costs, and different socio-economic groups have distinct resources to face these costs (Delbosc and Currie, 2011). A walkable environment with a walkable distance to the most important daily activities is the cheapest option and it reduces the need for longer distance trips. Moreover, it is well known that a walkable place has impact on other health determinants, such as physical activity and social interaction, that increase wellbeing (Loureiro et al., 2019).

Most previous studies about the relationship between walkable environment and socio-economic status were performed in the North American and Australian contexts, where the spatial distribution of socio-economic groups is stronger (Gullón et al., 2017). The European context has different development patterns with different spatial reasons to shape population distribution (Munoz, 2003). The Portuguese context, in this case, the LMA, shares these different developments where different socio-economic groups occupy different built environment characteristics across the city, with different levels of accessibility (Santana, 2007).

The design of a walkable environment that supports the use of active and public transport modes to the most important destinations allows the reduction of inequalities, promoting access by inexpensive modes of transportation (Badland et al., 2014; Giles-Corti et al., 2016). A built environment that promotes walking and cycling helps to reduce inequities and can benefit such aspects as health, traffic management, air quality and economy. Urban areas that reduce the need of mandatory transportation far from walkable distances can improve individual wellbeing and living conditions (Giles-Corti et al., 
2016). Unfortunately, disadvantaged populations face disparities on walkability, safety and health, and also inequities in facilities and built environment conditions. The benefits of walking are especially important for disadvantaged groups and they have a higher cost for disadvantaged and vulnerable populations (Adkins et al., 2017). The identification of less walkable areas can improve the design of policy and planning interventions (King and Clarke, 2015).

A walkable built environment refers to how favourable the built environment is for walking, and it can be used to improve physical activity and active travel. Walkability is commonly defended as a measure which defines whether the existing neighbourhood qualities encourage people to walk (Wang and Yang, 2019). The most used walkability indexes are composed of such built environment dimensions as residential density, intersection density, the retail floor area ratio and land use mix. The indicators follow three main categories ('three Ds'): density, diversity and design as determinants of walking. Later, these were expanded to 'five Ds' to include destination accessibility and distance to transit (Ewing and Cervero, 2010), which some authors claim can be conceived not as pure built environment but as (dependent) accessibility indicators, reframing the 'five Ds' as ' 3 Ds + A' (Vale and Pereira, 2016).

Pedestrian accessibility conditions of the residential neighbourhood define several aspects of daily life with impacts on wellbeing (Delbosc and Currie, 2011). One of these aspects is time spent on travelling, which reduces the free time available for other important activities, such as social interactions, family time and leisure (Lachapelle et al., 2016). In addition, the absence of free time is one of the major determinants of reduced social interaction. This shortage of free time is called 'time poverty', and can contribute to increase social exclusion and to reduce subjective wellbeing (Currie and Delbosc, 2010). Public transport accessibility is especially important for the disadvantaged population, more dependent on these mobility options (Lucas, 2012).

Urban green areas contribute to wellbeing by improving social interaction and integration (Picavet et al., 2016; Ward Thompson et al., 2016). Some interesting facts are the different effects on mental health and stress levels that urban green areas have in different socio-economic groups, and also the contribution of urban green areas to reducing economic health inequalities (Ward Thompson et al., 2016). A consensual result in the literature highlights the effects of urban green areas on health, not only in terms of physical activity but also in terms of mental health (Douglas et al., 2017; Loureiro et al., 2019; Picavet et al., 2016). Moreover, proximity to green areas is considered relevant for a walkable environment and contributes to better living conditions and healthy environments (Picavet et al., 2016). The contribution of green area accessibility to wellbeing is supported by literature which points to the influence on individual behaviours such as physical activity and social interaction. 
A walkable environment has been shown to influence property value (Carr et al., 2010). One of the most used walkability indicators, the Walk Score $\AA$, has been used to evaluate house prices, reflecting the impact of walkability on increasing this price and, consequently, influencing the socio-economic groups' location (Carr et al., 2010; Vale et al., 2016).

Property value has been used as a measure of socio-economic characteristics (Drewnowski et al., 2014; Jiao et al., 2016), due to the role of property value to conditionate the type of SES present in a place. Property value emphasizes the perception of poverty or wealth of a place. The individuals' perception of the surrounding built environment has an impact in their own view of SES, but it also has an impact on property values (Drewnowski et al., 2014). These aspects reinforce the impact of the built environment to the spatial distribution of different socio-economic groups, with property value being a condition for housing access. Accessibility to the city centre shapes individuals' home location, which results from the competition between several activities. The higher property values in the city centre exclude the most deprived population, yet the sprawl effects in modern development create settlements of higher SES far from the city centre (Koschinsky and Talen, 2015). Nevertheless, the distribution of population differs from city to city, and the occupation of a particular place by different socio-economic groups can change through time (Gullón et al., 2017). Furthermore, the spatial distribution of these places across countries and cities is not equal, and it is not always representative of the population status present in these places.

\subsection{METHODOLOGY}

\subsubsection{Study Area and Design}

The LMA is a regional administrative division composed of 18 municipalities and 211 civil parishes (34 937 census blocks). The LMA represents 3.3 per cent of the total area of Portugal, with approximately 3 million inhabitants $(25$ per cent of the Portuguese population). In economic terms, it represents more than 36 per cent of the national gross domestic product. The built environment was measured considering the walkability concept, including accessibility measures and topography conditions. The SES evaluation uses indicators of population wealth.

The relationship between walkability and SES was evaluated by a Spearman correlation matrix. Due to different urban and demographic contexts existent in LMA, four groups of census tracts were created considering 15 minutes, 30 minutes, 45 minutes and more than 45 minutes morning peak travel times from city centre (see Figure 3.1c in the 'Results' section). Travel time was calculated for car mode considering traffic information. The selection of 15 
minutes groups was supported by the impact of the commuting time for wellbeing, which is one of the major reasons for travel mode selection (Delbosc and Currie, 2011; Giles-Corti et al., 2016). Linear regression was performed for each group separately. We have analysed relationships between SES and walkability indicators and for the two indexes created.

\subsubsection{SES Measures}

The most common SES indexes use indicators such as income, education or occupation (Cebrecos et al., 2018; Santana et al., 2015). However, property value is important in shaping several aspects of health and wellbeing (Drewnowski et al., 2014; Jiao et al., 2016). In this chapter, we calculated an SES index that is composed of four indicators: (1) literacy rate; (2) employment rate; (3) percentage of households with a toilet; and (4) property value $(€ /$ $\mathrm{m}^{2}$ ). The indicators were standardized, and the z-scores were summed to form the composite SES index (Santana et al., 2015):

SES Index $=z$-score LiteracyRate $+z$-score EmploymentRate $+z$-scoreHouse-

WithToilet $+z$-score PropertyValue

Property value (euros $/ \mathrm{m}^{2}$ ) was obtained from a retail housing website (www imovirtual.com), collecting the coordinates of the real estate property for sale in November 2018. Based on the asking price and dimensions of the house, the price per square metre was calculated for each point. The points obtained were used to interpolate a surface using Empirical Bayesian Kriging available in ArcGIS 10.6 and predicting the property value to the census block centroids $\left(\right.$ euros $\left./ \mathrm{m}^{2}\right)$. The results for census block were then aggregated at census tract with an average value. The source of the remaining SES indicators is the 2011 census data aggregated at census tracts.

\subsubsection{Walkability Measure}

Walkability was measured for a $500 \mathrm{~m}$ buffer around each urban census block. The indicators were then aggregated at the census tract to better describe the bespoke neighbourhood and to control the modifiable area unit problem (Openshaw, 1983). We have calculated a walkability index in line with the most used walkability indexes (Wang and Yang, 2019), including density, diversity and design, but also comprising accessibility measures and topography conditions. Density was measured by household density based on the 2011 census data. Diversity was measured by the number of different types of activities obtained from points of interest information (POIs) from the Navteq ${ }^{1}$ database. The design dimension was measured by density of road intersections 
(nodes) with three or more links, also from the Navteq database. Topography was evaluated by the percentage of census block with a slope less than 8 per cent, calculated based on a digital terrain model raster with 30 metres resolution. Accessibility measures evaluate the accessibility to green areas and public transport accessibility. The green areas considered were 'green urban areas' and 'forests' classification in Urban Atlas (Kabisch and Haase, 2014). Two green areas indicators were created: the percentage of the buffer area occupied by green area and the total of green area in the census block.

Public transport accessibility was measured following Currie and Delbosc's (2010) methodology, adapted to the LMA context by considering station buffers sizes of $400 \mathrm{~m}$ to bus, $800 \mathrm{~m}$ to train, $500 \mathrm{~m}$ to tram and $800 \mathrm{~m}$ to ferryboat. The public transport index consists of daily transport supply in each walking buffer, which is calculated by the proportion of overlapped buffers in the census tract. The calculation was performed with BetterBusBuffers, an ArcGIS tool based on a General Transit Feed Specification (GTFS) database available at the city's open data portal. ${ }^{2}$ The final walkability index is given by the formula:

Walkability Index $=$ z-score Density + z-score Diversity + z-score Design + z-score Green Area + z-score Slope + z-score PT Accessibility

\subsection{RESULTS}

Figure 3.1 illustrates the spatial pattern of the SES and walkability index. The SES index does not reveal a clear pattern, having high and low values in the four census tract groups. The SES map highlights the spatial pattern of property values, particularly clear for Lisbon's city centre and coastal line, which have higher property values. In the case of the walkability index, a clear distinction is visible between Lisbon's city centre and the rest of the LMA. The 15 minutes census tract group and Lisbon municipality have the highest walkability index values. The walkability index emphasizes the city centre with the highest public transport offer, but also highlights the census tracts further from the city centre, which have lower accessibility but possess higher levels of green areas and higher percentages of low slope area.

The results of the Spearman correlation (Figure 3.2) show no association between socio-economic indicators and walkability indicators (grey box). Property value is the socio-economic indicator with the highest correlation values with walkability indicators. Particularly, accessibility to public transport shows a significant relation with property value, although the value is small (0.53). The variety dimension $(0.48)$ and walkability index $(0.46)$ are also significantly related to property values, but with lower coefficients. The 


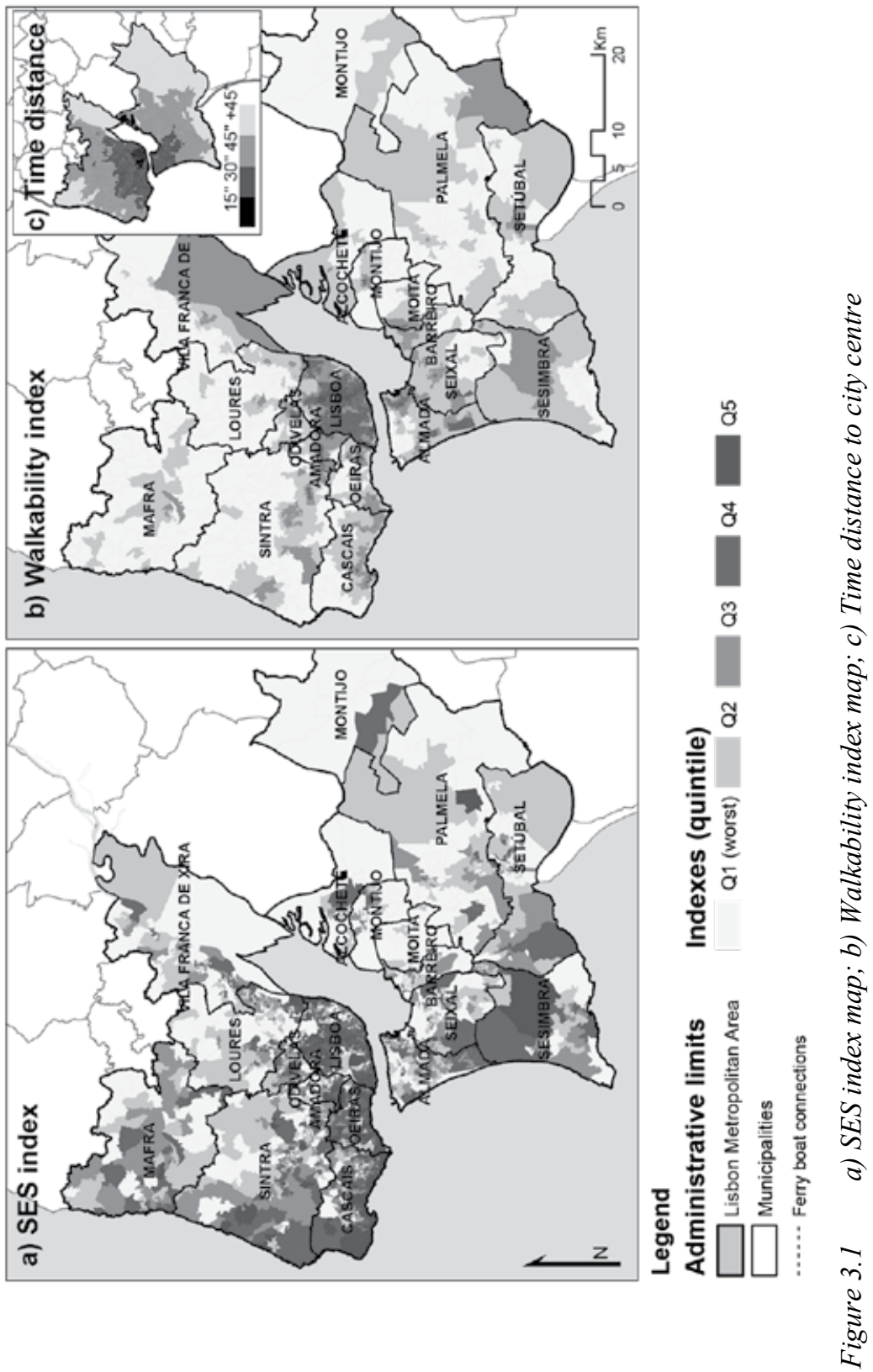




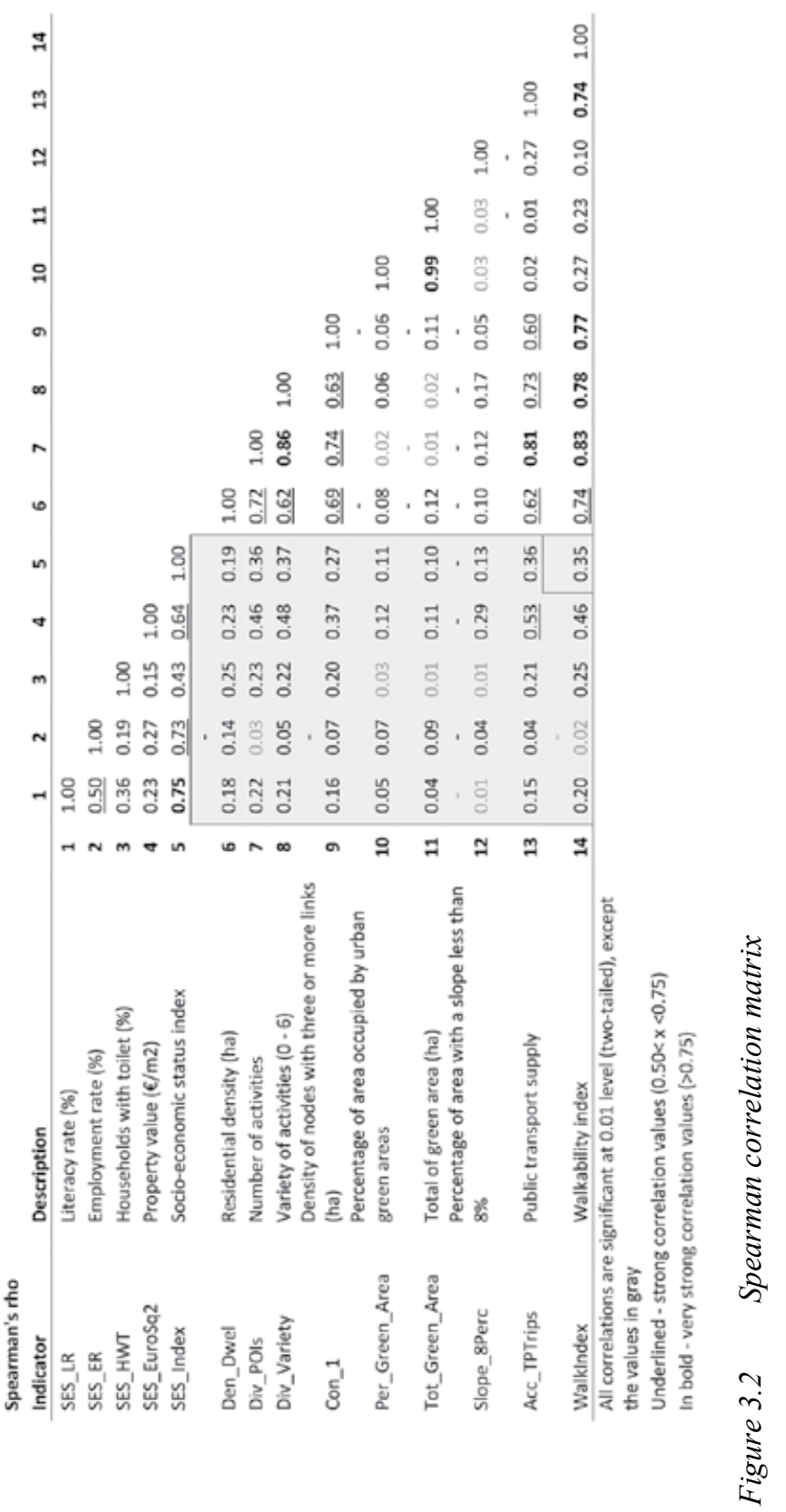


highest values are observed between the different built environment indicators and the walkability index; for example, walkability index and variety of activities (0.86), and the walkability index and public transport supply (0.74).

Figure 3.3 consists of scatter plots which show the relationship between walkability indicators and socio-economic indicators for the four groups of census tracts considered. Considering the entire LMA, the highest regression value is between the walkability index and property value $\left(\mathrm{r}^{2}=0.32\right)$. The highest walkability indicator value correlated with the SES index is public transport supply $\left(\mathrm{r}^{2}=0.13\right)$, followed by diversity $\left(\mathrm{r}^{2}=12\right)$ and the walkability index $\left(r^{2}=0.12\right)$. The results stress the importance of these components for the indexes created. The remaining walkability indicators do not reveal a correlation with the SES index.

Regression values for the four groups are low. However, the difference between the closest group, at 15 minutes from the city centre, and the rest of the LMA are clear. The highest correlation value is diversity $\left(r^{2}=0.16\right)$ for the 15 minutes group, and the following group has a much lower correlation value $\left(r^{2}=0.03\right)$. The following highest correlation is between the walkability index and property value for the 15 minutes group $\left(r^{2}=0.13\right)$. In this case, the difference for the next group is lower $\left(r^{2}=0.11\right)$. The slope and SES index have a high value for the 15 minutes group $\left(r^{2}=0.12\right)$, but a very low value for the rest of the groups. The walkability index and the SES index have the following highest value for the 15 minutes group $\left(r^{2}=0.09\right)$. For the next group, the difference is not as high $\left(\mathrm{r}^{2}=0.05\right)$. Public transport supply, green area and design have a similar behaviour for correlation values, stressing the difference between the closest census tracts and the rest of the city. The density indicator reveals a lack of correlation with the SES index for all the four groups.

\subsection{CONCLUSIONS}

This chapter has explored the relationship between the distribution of the walkable qualities across different socio-economic groups, considering the distance to the city centre where most of the facilities are concentrated. The description of the built environment by walkability measures defines most of the qualities of a human scale city. The analysis of distribution of the human scale city qualities by the type of population served by these qualities can point towards policies and interventions to improve wellbeing by creating a walkable environment.

We have found a low correlation between socio-economic indicators and walkable indicators. The results reveal a relationship between property value and accessibility by walking and also by public transport, in line with other studies (Carr et al., 2010; Koster and Rouwendal, 2012). Correlation values are significantly different between the closer groups and the rest of the LMA, 


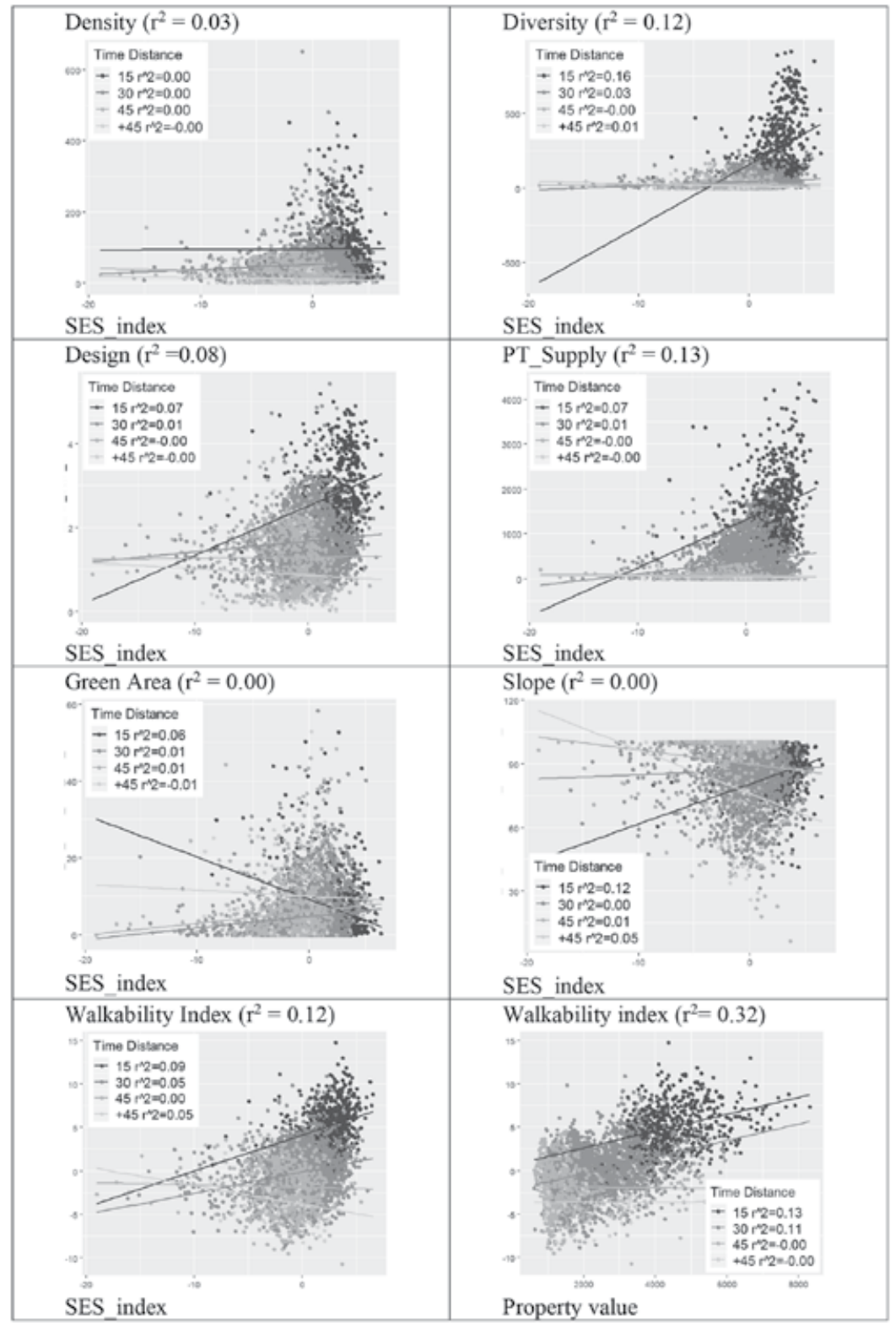

Figure $3.3 \quad$ Scatter plots for each walkability measure and SES index, by time distance to city centre 
especially for diversity of activities and public transport supply. The four groups remain with lower correlation values, but the closest group is significantly different. The highest correlation between the SES index and diversity identifies that the most central area is best served by public transport and activities. However, only 10 per cent of the LMA population lives within 15 minutes of the city centre. The major proportion of the population (63 per cent) lives in the second group ( 15 to 30 minutes) where a relatively good value of walkability was registered. The census tracts at more than 45 minutes from the city centre represent only 2 per cent of the LMA population, with the lowest walkability measures. The lower correlation and regression values between walkability and SES reveal that the most deprived people occupy places with different walkability qualities. Nonetheless, it seems that the city centre is mostly an exclusive area, occupied by the highest SES.

The results point to a relatively good distribution of the walkability qualities in the LMA, though an important distinction exists between the city centre and the rest of the LMA, especially in public transport supply and diversity of activities. Future studies should consider time distance to the centre in other modes, and different census tract groups should be tested. The chapter is a starting point to evaluate the distribution of walkability qualities in the LMA, pointing to the most human scale parts of the city that can contribute to better social inclusion.

\section{NOTES}

1. See https://www.here.com/navteq.

2. See http://lisboaaberta.cm-lisboa.pt/index.php/pt.

\section{REFERENCES}

Adkins, A., Makarewicz, C., Scanze, M., Ingram, M., and Luhr, G. (2017). Contextualizing walkability: do relationships between built environments and walking vary by socioeconomic context? Journal of the American Planning Association, 83(3), 296-314.

Badland, H., Whitzman, C., Lowe, M., Davern, M., Aye, L., et al. (2014). Urban liveability: emerging lessons from Australia for exploring the potential for indicators to measure the social determinants of health. Social Science and Medicine, 111, 64-73.

Barton, H., and Grant, M. (2006). A health map for the local human habitat. Journal of the Royal Society for the Promotion of Health, 126(6), 252-253.

Carr, L.J., Dunsiger, S.I., and Marcus, B.H. (2010). Walk Score ${ }^{\mathrm{TM}}$ as a global estimate of neighborhood walkability. American Journal of Preventive Medicine, 39(5), 460-463.

Cebrecos, A., Domínguez-Berjón, M.F., Duque, I., Franco, M., and Escobar, F. (2018). Geographic and statistic stability of deprivation aggregated measures at different spatial units in health research. Applied Geography, 95, 9-18. 
Currie, G., and Delbosc, A. (2010). Modelling the social and psychological impacts of transport disadvantage. Transportation, 37(6), 953-966.

Delbosc, A., and Currie, G. (2011). The spatial context of transport disadvantage, social exclusion and well-being. Journal of Transport Geography, 19(6), 1130-1137.

Douglas, O., Lennon, M., and Scott, M. (2017). Green space benefits for health and well-being: a life-course approach for urban planning, design and management. Cities, 66, 53-62.

Drewnowski, A., Aggarwal, A., Rehm, C.D., Cohen-Cline, H., Hurvitz, P.M., and Moudon, A.V. (2014). Environments perceived as obesogenic have lower residential property values. American Journal of Preventive Medicine, 47(3), 260-274.

Ewing, R., and Cervero, R. (2010). Travel and the built environment - a meta-analysis. Journal of the American Planning Association, 76(3), 265-294.

Geurs, K.T., and van Wee, B. (2004). Accessibility evaluation of land-use and transport strategies: review and research directions. Journal of Transport Geography, 12(2), 127-140.

Gilderbloom, J.I., Riggs, W.W., and Meares, W.L. (2015). Does walkability matter? An examination of walkability's impact on housing values, foreclosures and crime. Cities, 42, 13-24.

Giles-Corti, B., Vernez-Moudon, A., Reis, R., Turrell, G., Dannenberg, A.L., et al. (2016). City planning and population health: a global challenge. Lancet, 388(10062), $1-13$.

Gullón, P., Bilal, U., Cebrecos, A., Badland, H.M., Galán, I., and Franco, M. (2017). Intersection of neighborhood dynamics and socioeconomic status in small-area walkability: the Heart Healthy Hoods project. International Journal of Health Geographics, 16(1), 21.

Jiao, J., Drewnowski, A., Moudon, A.V., Aggarwal, A., Oppert, J.-M., et al. (2016). The impact of area residential property values on self-rated health: a cross-sectional comparative study of Seattle and Paris. Preventive Medicine Reports, 4, 68-74.

Kabisch, N., and Haase, D. (2014). Green justice or just green? Provision of urban green spaces in Berlin, Germany. Landscape and Urban Planning, 122, 129-139.

King, K.E., and Clarke, P.J. (2015). A disadvantaged advantage in walkability: findings from socioeconomic and geographical analysis of national built environment data in the United States. American Journal of Epidemiology, 181(1), 17-25.

Koschinsky, J., and Talen, E. (2015). Affordable housing and walkable neighborhoods: a national urban analysis. Cityscape: A Journal of Policy Development and Research, 17(2), 13-56.

Koster, H., and Rouwendal, J. (2012). The impact of mixed land use on residential property values. Journal of Regional Science, 52(5), 733-761.

Lachapelle, U., Frank, L.D., Sallis, J.F., Saelens, B.E., and Conway, T.L. (2016). Active transportation by transit-dependent and choice riders and potential displacement of leisure physical activity. Journal of Planning Education and Research, $36(2), 225-238$.

Loureiro, A., Santana, P., Nunes, C., and Almendra, R. (2019). The role of individual and neighborhood characteristics on mental health after a period of economic crisis in the Lisbon region (Portugal): a multilevel analysis. International Journal of Environmental Research and Public Health, 16(15), 2647.

Lucas, K. (2012). Transport and social exclusion: where are we now? Transport Policy, $20,105-113$. 
Marmot, M., Allen, J., Bell, R., Bloomer, E., and Goldblatt, P. (2012). WHO European review of social determinants of health and the health divide. Lancet, 380(9846), 1011-1029.

Munoz, F. (2003). Lock living: urban sprawl in Mediterranean cities. Cities, 20(6), 381-385.

Openshaw, S. (1983). The Modifiable Areal Unit Problem. Concepts and Techniques in Modern Geography 38. Norwich: Geobooks.

Picavet, H.S.J., Milder, I., Kruize, H., de Vries, S., Hermans, T., and Wendel-Vos, W. (2016). Greener living environment healthier people? Preventive Medicine, 89, 7-14.

Santana, P. (ed.). (2007). A Cidade e a Saúde. Coimbra: Edições Almedina.

Santana, P., Costa, C., Marí-Dell'Olmo, M., Gotsens, M., and Borrell, C. (2015). Mortality, material deprivation and urbanization: exploring the social patterns of a metropolitan area. International Journal for Equity in Health, 14(1), 1-13.

Vale, D.S., and Pereira, M. (2016). Influence on pedestrian commuting behavior of the built environment surrounding destinations: a structural equations modeling approach. International Journal of Sustainable Transportation, 10(8), 730-741.

Vale, D.S., Saraiva, M., and Pereira, M. (2016). Active accessibility: a review of operational measures of walking and cycling accessibility. Journal of Transport and Land Use, 9(1), 209-235.

Wang, H., and Yang, Y. (2019). Neighbourhood walkability: a review and bibliometric analysis. Cities, 93, 43-61.

Ward Thompson, C., Aspinall, P., Roe, J., Robertson, L., and Miller, D. (2016). Mitigating stress and supporting health in deprived urban communities: the importance of green space and the social environment. International Journal of Environmental Research and Public Health, 13(4), 440. https://doi.org/10.3390/ ijerph13040440. 